\title{
Distributed Data and Job Management for the XENON1T Experiment
}

\author{
Benedikt Riedel $^{*}$ \\ Enrico Fermi Institute, University of \\ Chicago \\ Chicago, Illinois \\ briedel@uchicago.edu \\ Jan Conrad \\ Oskar Klein Centre, Department of \\ Physics, Stockholm University \\ Stockholm, Sweden \\ conrad@fysik.su.se \\ Luca Grandi \\ Department of Physics and Kavli \\ Institute for Cosmological Physics, \\ University of Chicago \\ Chicago, Illinois \\ lgrandi@uchicago.edu \\ Gabriella Sartorelli \\ Department of Physics and \\ Astronomy, University of Bologna \\ and INFN-Bologna \\ Bologna, Italy \\ gabriella.sartorelli@bo.infn.it \\ Judith Stephen \\ Enrico Fermi Institute, University of \\ Chicago \\ Chicago, Illinois \\ jlstephen@uchicago.edu
}

\author{
Boris Bauermeister \\ Oskar Klein Centre, Department of \\ Physics, Stockholm University \\ Stockholm, Sweden \\ Boris.Bauermeister@fysik.su.se \\ Patrick de Perio \\ Physics Department, Columbia \\ University \\ New York, New York \\ pdeperio@astro.columbia.edu
}

Francesco Lombardi

Department of Physics, University of

California San Diego

La Jolla, California

flombardi@physics.ucsd.edu

Marco Selvi

Department of Physics and

Astronomy, University of Bologna

and INFN-Bologna

Bologna, Italy

Marco.Selvi@bo.infn.it

Suchandra Thapa

Enrico Fermi Institute, University of Chicago

Chicago, Illinois

ssthapa@uchicago.edu

\author{
Lincoln Bryant \\ Enrico Fermi Institute, University of \\ Chicago \\ Chicago, Illinois \\ lincolnb@uchicago.edu \\ Robert W. Gardner \\ Enrico Fermi Institute, University of \\ Chicago \\ Chicago, Illinois \\ rwg@uchicago.edu \\ Alfio Rizzo \\ Physics Department, Columbia \\ University \\ New York, New York \\ rizzo.alfio@gmail.com
}

Evan Shockley

Department of Physics and Kavli

Institute for Cosmological Physics,

University of Chicago

Chicago, Illinois

ershockley@uchicago.edu

Christopher Tunnell

Department of Physics and Kavli

Institute for Cosmological Physics,

University of Chicago

Chicago, Illinois

tunnell@uchicago.edu

\begin{abstract}
We present the distributed data and job management scheme on the Open Science Grid (OSG) and European Grid Infrastructure (EGI) that was developed for the XENON1T experiment. The experiment aims to detect dark matter using the first ton-scale detector of this kind, a 2000-kg-target-mass dual-phase (liquid-gas) xenon

${ }^{*}$ Corresponding Author

Permission to make digital or hard copies of all or part of this work for personal or classroom use is granted without fee provided that copies are not made or distributed for profit or commercial advantage and that copies bear this notice and the full citation on the first page. Copyrights for components of this work owned by others than ACM must be honored. Abstracting with credit is permitted. To copy otherwise, or republish, to post on servers or to redistribute to lists, requires prior specific permission and/or a fee. Request permissions from permissions@acm.org.

PEARC '18, fuly 22-26, 2018, Pittsburgh, PA, USA

(C) 2018 Association for Computing Machinery.

ACM ISBN 978-1-4503-6446-1/18/07 ...\$15.00

https://doi.org/10.1145/3219104.3219155
\end{abstract}

time projection chamber in operation at the Laboratori Nazionali del Gran Sasso in Italy. The experiment's data is automatically spread across several sites on EGI and OSG. The data location and movement is managed using the rucio software package developed by the ATLAS collaboration. The job submission to OSG, EGI, SDSC's Comet Supercomputer, and campus HPC resources is done through the $\mathrm{CI}$ Connect infrastructure that is directly connected to the general OSG infrastructure. This paper will describe the overall integration of distributed storage and compute resources into a common interface, respectively, and integration into the existing XENON1T software

\section{CCS CONCEPTS}

- Computer systems organization $\rightarrow$ Grid computing; 


\section{KEYWORDS}

OSG, distributed data access, CVMFS, XENON, EGI, XENON1T, XSEDE, Campus Cluster, HTCondor, Distributed computing, Grid computing

\section{ACM Reference Format:}

Benedikt Riedel, Boris Bauermeister, Lincoln Bryant, Jan Conrad, Patrick de Perio, Robert W. Gardner, Luca Grandi, Francesco Lombardi, Alfio Rizzo, Gabriella Sartorelli, Marco Selvi, Evan Shockley, Judith Stephen, Suchandra Thapa, and Christopher Tunnell. 2018. Distributed Data and Job Management for the XENON1T Experiment. In Proceedings of Practice and Experience in Advanced Research Computing (PEARC '18). ACM, New York, NY, USA, 8 pages. https://doi.org/10.1145/3219104.3219155

\section{INTRODUCTION}

The matter composition of the universe has been a debated topic among scientists for centuries. In the last couple of decades a series of astronomical and astrophysical measurements have corroborated the hypothesis that ordinary matter, e.g. electrons, quarks, neutrinos, etc., represents only $15 \%$ of the total matter in the universe $[11,12,30,35]$. The remaining $85 \%$ is thought to be made of a new, yet-undiscovered exotic species of elementary particles called dark matter [11, 12, 30, 35]. These indirect evidences of its existence triggered a world-wide effort to try observe its interaction with ordinary matter in extremely sensitive detectors, but its nature is still a mystery.

The XENON experimental program $[15,16,48]$ is searching for weakly interacting massive particles (WIMPs) [34], hypothetical particles that, if existing, could account for dark matter and that might interact with ordinary matter through nuclear recoil. XENON1T is the third and current generation of the experimental program. The experiment employs a dual-phase (liquid-gas) xenon time projection chamber (TPC) featuring two tonnes of ultrapure liquid xenon as target for WIMPs. The specialized detector is designed to be sensitive to rare nuclear recoils of xenon nuclei, which could possibly be induced by WIMPs scattering within the detector. The TPC is surrounded by a water-based muon veto (MV). Each sub-detector is read out by its own data acquisition system (DAQ). The detector is located underground at the Laboratori Nazionali del Gran Sasso (LNGS) in Italy to shield the experiment from cosmic rays.

XENON1T is an order of magnitude larger than any of its predecessor experiments. This upscaling in detector size produced a proportionate increase in the data rate and computing needs of the collaboration. The size of the data set required the collaboration to transition from a centralized computing model, i.e. the entire dataset is stored on a local facility at various institutions, to having to distribute the data across collaboration resources. Similarly, the computing requirements called for incorporating distributed resources, such as the Open Science Grid (OSG) [41] and the European Grid Infrastructure (EGI) [37], for main computing tasks, e.g initial data processing and Monte Carlo production.

In this paper, we will describe the data and job management workflow for the XENON1T experiment; specifically, how we created a common interfaces for both storage and job management. For the storage interface, we used the rucio data management system [19] from the ATLAS experiment [25]. The job management interface uses HTCondor [38, 44, 45], OSG's glideinWMS [43], and beta access to the Virtual Clusters for Computing (VC3) [22] project to tie the computing resources, e.g. campus clusters, Extreme Science and Engineering Discovery Environment (XSEDE) resources [40, 46], and national computing resources, together using the OSG infrastructure. For a visual overview of the system, see Figure 1.

\section{DATA MANAGEMENT}

The significant increase in the data rate compared to previous generations of the XENON experiment required re-engineering the data management. From a data rate perspective, the detector operations can be split into two modes: "Background" and "Calibration." In background mode, the detector collects roughly $1.3 \mathrm{~TB}$ per day. During calibration periods the detector rate increases significantly and can reach 13 TB per day, depending on the type of calibration that is being performed. A selection of calibration sources and their rates is given in Table 1.

A significant challenge for the collaboration has been that there is no single institution that has the capacity to store the entire data set. This requires that the data to either be stored in a cloud environment or be distributed across various collaboration institutions. Storing the data in a cloud environment is prohibitively expensive at this point. The data set size and the network traffic charges would consume the entire computing budget several times over.

The only feasible option was to distribute the data across several computing facilities associated with collaboration institutions. Initially, we considered creating our own data management system using Globus Connect [14,32] to transfer the data between sites. Globus Connect would have acted as a transfer execution and monitoring service. We could have used the existing infrastructure to track the location of files and existing tools to interact with Globus Connect. Due to the storage architectures in use at some of our computing facilities we decided to drop Globus in favor of GridFTP [23] or Xrootd [8, 20, 31].

For the initial operations of the experiment, the collaboration used the Python-based tool Copying All XENON1T (cax) to provide the database API, data management, and job management software. cax used the Python-library paramiko [2] to transfer the data between sites using scp [4]. The transfer action was controlled through the run status and settings in the central XENON database called the runsDB. Besides the run status, the runsDB stores detector settings, triggers settings, DAQ settings, etc.. Once a data-taking increment, also known as a "run", was complete, the DAQ would change the status of the run in the runsDB such that the data transfer would be initiated. At the end of the data transfer, the runsDB was updated to reflect the success or failure of a run as well as its location in case the transfer succeeded.

cax's data transfer feature was sufficient for the first few months of detector operation during which a single storage location could be used. Other storage locations are large computing sites for the Large Hadron Collider (LHC) experiments, i.e. Tier 1 and Tier 2 sites [1]. These sites require the use of GridFTP or Xrootd for large scale file transfers. To use the allocated storage at the Tier 1 and Tier 2 sites, XENON adopted rucio as their data management system. rucio has been developed as the data management software for the ATLAS experiment at the LHC. It has been thoroughly tested in 


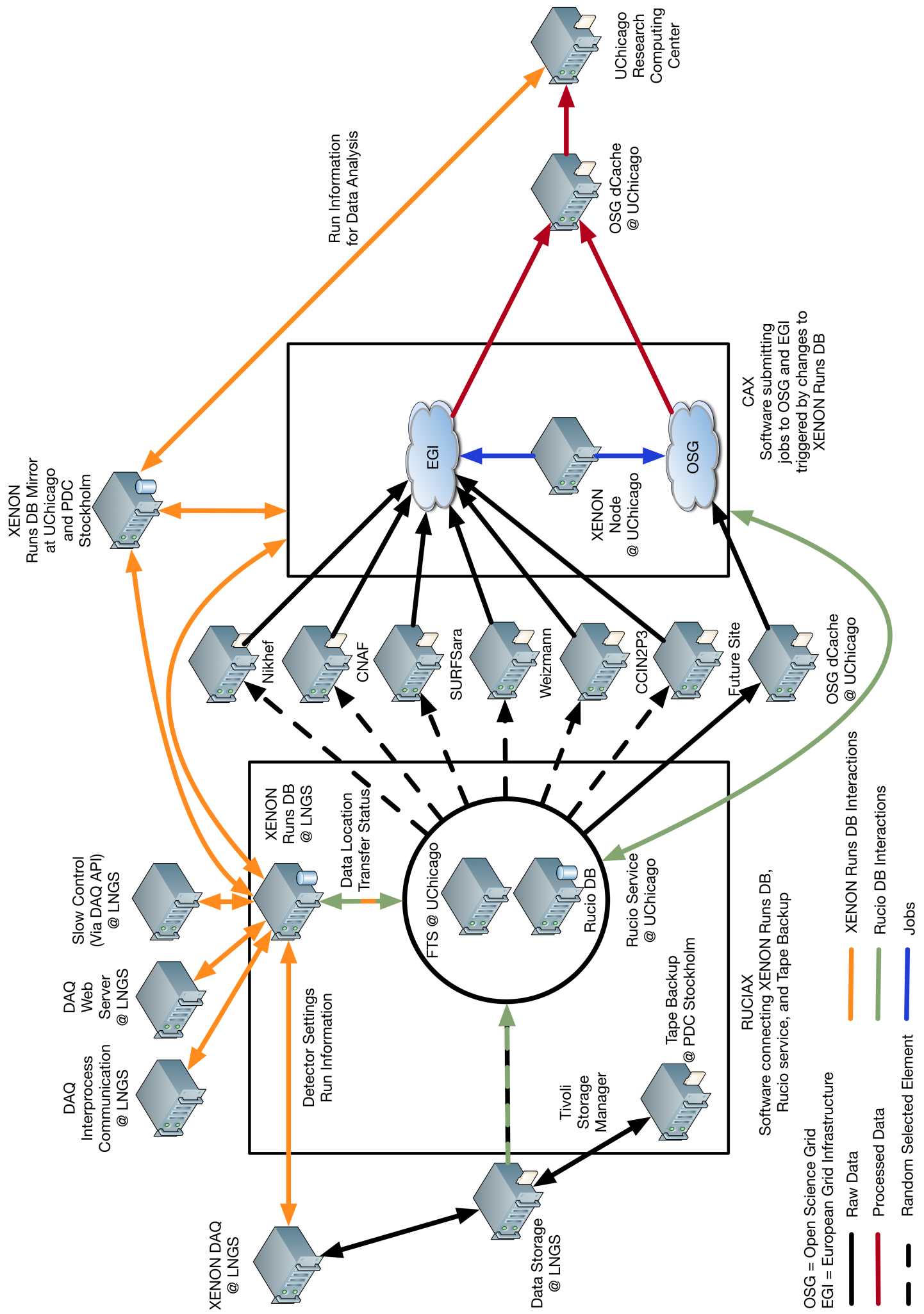

Figure 1: Overview of the XENON1T Job and Data Management Scheme. 
Table 1: Summary of Storage Requirements for XENON1T

\begin{tabular}{c|c|c|c}
\hline Source & Size per Event $(\mathrm{MB})$ & Total Events per Year $\left(\times 10^{6}\right)$ & Storage (PB/Year) \\
\hline${ }^{\text {Background }}$ & 2.01 & 139 & 0.279 \\
${ }^{83 \mathrm{~m}} \mathrm{Kr}$ & 0.78 & 66 & 0.051 \\
${ }^{220} \mathrm{Rn}$ & 0.67 & 145 & 0.097 \\
Neutron Generator & 2.71 & 4.5 & 0.013 \\
LED & 0.12 & 192 & 0.023 \\
\hline Total & & 546.5 & 0.463
\end{tabular}

Table 2: Overall Storage Usage for XENON1T as of March 26, 2018

\begin{tabular}{c|c|c|c}
\hline Storage Location & Storage Region & Used Storage (TB) & Allocated Storage (TB) \\
\hline OSG dCache & OSG & 441.5 & 450 \\
SurfSARA & EGI & 43.9 & 500 \\
Nikhef & EGI & 239.9 & 451 \\
CCIN2P3 & EGI & 236.9 & 400 \\
Weizmann Institute & EGI & 80 & 80 \\
INFN/CNAF & EGI & 23 & 94 \\
\hline Total & & 1065.2 & 1975
\end{tabular}

Table 3: Overall Number of Files and Datasets Usage as of March 26, 2018

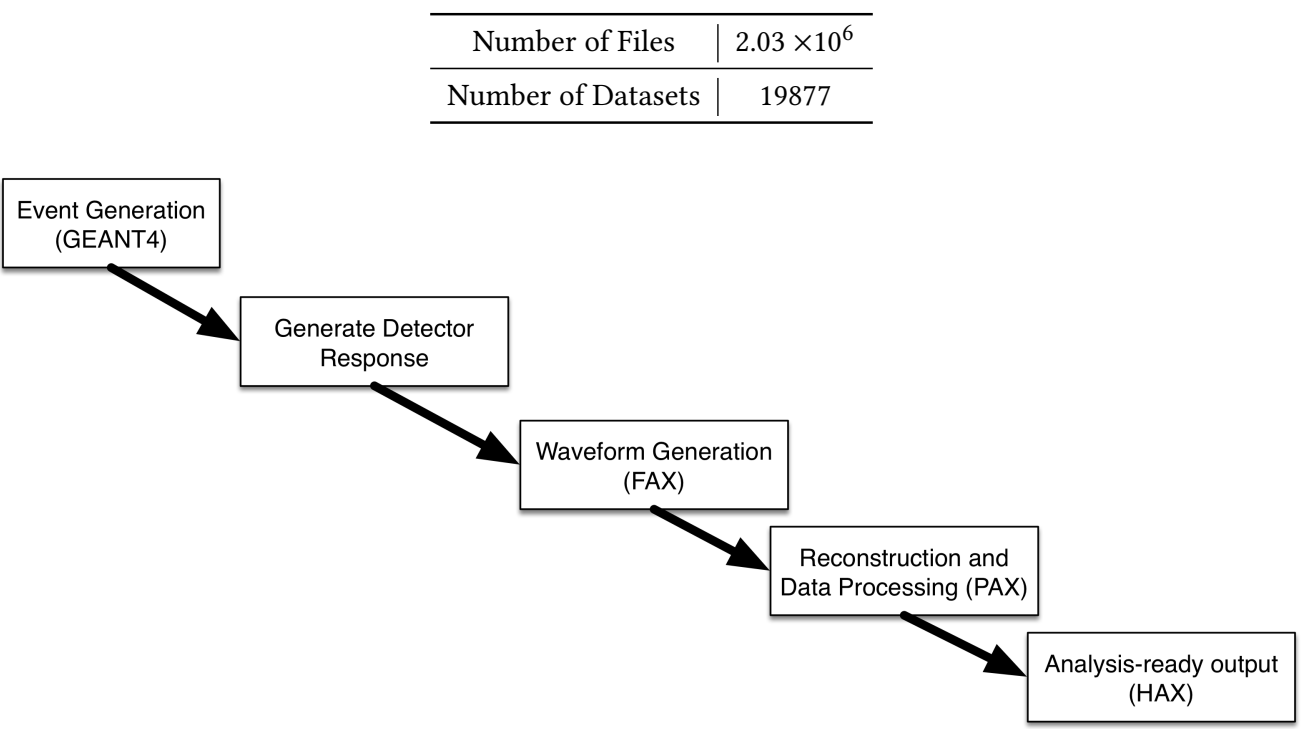

Figure 2: Overview of the XENON1T Monte Carlo Workflow [27]

a much more demanding environment as well as with much larger data volume.

Adopting rucio brought significant integration work with it. One of the major tasks was decoupling data movement from cax.
We developed a tool named ruciax that provides an interface between the rucio data management service and cax's runsDB interface.

The adoption process was performed in close collaboration with the rucio developers. This encouraged improvements and changes 
to rucio that provide more flexibility and better support for experiments besides ATLAS and Alpha Magnetic Spectrometer (AMS) [18, 36]. The adoption of rucio by XENON1T and AMS has spawned efforts by several other experiments, mainly CMS [26], LIGO [10], and IceCube [9], to evaluate rucio for their data management needs. This has led to greater community effort to produce a generalpurpose data management system.

The ruciax workflow differs significantly from the original cax data management workflow. Instead of controlling data placement and movement, ruciax delegates those tasks to rucio. In the ruciax workflow, once a run has been declared complete by the DAQ, ruciax will add the files to the rucio catalog. ruciax then adds a "transfer rule", a data placement directive in rucio, to move the data to two storage locations, one in Europe and one in the US. In addition, ruciax is used to overcome the difference in minimum Python versions required, i.e. 2.6 vs. 3.4, between rucio and cax.

There are five storage sites in Europe and one site in the US, see Table 2. These sites provide storage in form of tape, disk, or a combination of the two as well as a variety of different storage architectures, e.g. dCache [39], DPM [33], and GPFS [42]. The selection process of the European sites has evolved over time. Initially, we used a storage endpoint until the storage allocation was exhausted. Due to overloading a number of storage endpoints during large processing campaigns, we switched to randomly selecting a European site with available storage. This reduced the strain on the individual endpoints and allowed for smoother completion of large processing campaigns.

To ensure that the data is backed up, the collaboration has a tape storage allocation at the Parallelldatorcentrum (PDC) in Stockholm [3]. This tape storage can only be accessed through Tivoli Storage Manager (TSM) [5] and is therefore completely separate from the rucio deployment. To connect the runsDB and TSM, we deployed a specialized version of cax, called cax-tsm. cax-tsm communicates with TSM via command line interface and the updates to the runsDB once the data has been successfully uploaded.

For an overview of the usage statistics of XENON1T's rucio instance, see Table 2 and 3. Overall the adoption of rucio by XENON1T has been a great success. We were able to tie together the collaboration's storage allocations across OSG and EGI into a single interface and have been able to simplify the data management process.

\section{JOB MANAGEMENT}

The job management for XENON1T is split between two primary computing tasks: Monte Carlo production and data processing. For both tasks, the bulk of the work relies on submitting jobs to the OSG and EGI through the OSG infrastructure at the University of Chicago. To integrate the EGI sites for XENON1T, the sites were added to the OSG glideinWMS infrastructure. To simplify adding new sites, a XENON-specific authentication certificate was added to glideinWMS.

In addition to integrating EGI resources through the OSG interface, we have also added the NSF Supercomputer Comet at the San Diego Supercomputing Center [40,47] to the XENON resource pool via the OSG interface. Specifically, this was done through the OSG interface at Comet. Using the VC3 project, we added the University of Chicago's Research Computing Center (RCC) [6] to the resource pool as well.

The Monte Carlo production workflow allows a full detector simulation to determine the response to calibration sources or WIMPs, see Figure 2. The workflow starts at generating particle interactions and propagating products in GEANT4 [13]. From this point, we are using internal XENON code to simulate the detector response, i.e. which PMTs would be triggered and how much signal they would see, waveform generation [27], and various levels of data analysis, see reconstruction and analysis-ready output steps. This entire chain is controlled and managed using the Pegasus workflow management system [29]. The workflows are triggered by individual users. There has yet to be a need to automate the submission of Monte Carlo workloads. This is mostly due to having a limited number of permutations of the needed Monte Carlo datasets.

The submission of data processing workloads is a much more complicated workflow. This is partially self-inflicted and partially due to the iterative nature of the data analysis process. In general, the job submission is handled through cax. Once the data for a run has been transferred successfully, cax will submit jobs that determine the PMT correction factors to the Midway1 HPC cluster located at RCC. The jobs cannot be run on OSG or EGI because a host or set of hosts must be whitelisted to allow write access to the runsDB.

Once these correction factors have been written to the runsDB, an HTCondor DAGMan [28] job is submitted to the XENON CI Connect [7] instance at University of Chicago. The DAGMan workflow contains the following steps:

(1) Fetch run configuration from the runsDB as a JSON file

(2) Submit one job per file per run

(3) Merge output files from jobs into a single file

(4) Transfer data to UChicago RCC

If all steps are successful, the run will be marked as processed in the runsDB.

After significant updates to the analysis software, pax, the entire data set must be "re-processed" to reflect improvements in detector understanding as well as software/algorithm performance. These reprocessing campaigns typically occurred every few months, with the rate decreasing with the experiment's maturity. The reprocessing steps are the same as the data processing steps modulo the number of files that have to be processed and the version of the processing software used.

Overall, the current setup for data processing and Monte Carlo has been extraordinarily successful. Through the OSG infrastructure and support, XENON was able to transition its entire Monte Carlo production and general data processing from RCC to OSG and EGI. The collaboration consumed over 9 million hours across allocated and opportunistic resources that included grid facilities, NSF supercomputers, and campus clusters, see Figure 3.

\section{SOFTWARE MANAGEMENT}

Software management for data processing and Monte Carlo production is handled through deployHQ [17] for the automatic deployment and CernVM-FS (CVMFS) [21,24] for distribution of the software across OSG and EGI. deployHQ is used to automatically deploy the newest version and releases of the XENON processing 


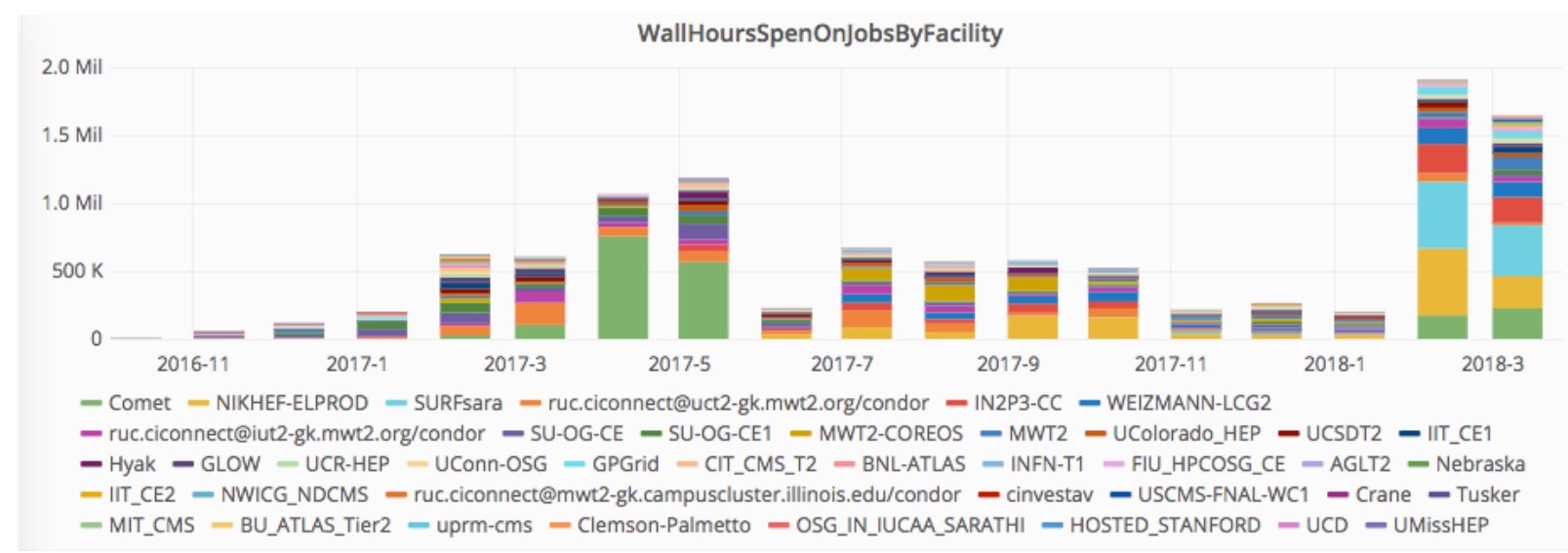

Figure 3: Summary of XENON1T's usage across OSG, EGI, NSF supercomputers and campus clusters on a per month basis

Table 4: Summary of estimated Storage Requirements for XENONnT

\begin{tabular}{c|c|c|c}
\hline Source & Size per Event $(\mathrm{MB})$ & Total Events per Year $\left(\times 10^{6}\right)$ & Storage (PB/Year) \\
\hline Background & 3.62 & 278 & 0.502 \\
${ }^{83 \mathrm{~m}} \mathrm{Kr}$ & 1.40 & 198 & 0.277 \\
${ }^{220} \mathrm{Rn}$ & 1.21 & $145-436$ & $0.194-0.528$ \\
Neutron Generator & 1.21 & $4.5-135$ & $0.054-0.164$ \\
LED & 0.24 & 192 & 0.046 \\
\hline Total & & $817.5-1239$ & $1.073-1.517$
\end{tabular}

software and data analysis software on both RCC and CVMFS. This allows for rapid testing of the newest version of the code, if needed. Distributing software to OSG and EGI sites via CVMFS is fairly standard for experiments using the OSG. It provides easy means to distribute software throughout grid sites.

\section{FUTURE-XENONNT}

The planning and initial implementation of the data and job management for the next generation experiment, XENONnT, has already begun. The experiment is scheduled to start taking data in mid-2019. The current plan is the increase the TPC volume to $8000 \mathrm{~kg}$ of liquid xenon. The new experimental setup will also have an additional veto layer called the Neutron Veto (NV).

The larger detector will require modifications to the current data and job management. The processing chain and its products will undergo significant changes. The larger estimated data volume, see Table 4, and improved knowledge about data access patterns has informed changes to the data organization. Rather than store the full raw dataset for later re-processing, the data coming from the detector will be filtered to only include interesting events. The full raw dataset will only be stored on tape at one or two sites, where one of these sites is for long-term archival. The filtered raw dataset will be stored at OSG/EGI sites for later reprocessing. The overall data volume of the reduced dataset will be similar to the current data volume of XENON1T.

The XENON collaboration has decided to continue using rucio for their data management needs. There will be data types added to the rucio catalog. We are considering having rucio also manage the processed data, as only a small fraction of users $(<10 \%)$ directly interact with the processed data. A typical analyst interacts with a even more refined data product called "minitrees" produced by another analysis toolkit, named hax [27]. This will help reduce the disk usage at the main analysis facility and help move more of the processing to the distributed resources.

Job management will be modified to accommodate the changes to the data management and improve access to distributed computing resources. This means that there will be an additional step in the processing chain and the final destination of processed data will not be at RCC. The calculation of the corrections that are currently done at RCC will also be moved to grid computing resources.

These changes to data and job management will require changes to cax and ruciax. We will separate the various interfaces, i.e. runsDB interface, batch system interface, etc., into individual APIs. In particular, the runsDB interface will be transitioned to a RESTful API to allow for better isolation of the database and better load balancing. The motivation behind the transition to separate APIs is to reduce the maintenance burden on any given individual and to allow experts to create and maintain the individual APIs. Additionally, having separate APIs will reduce the need for compatibility with certain versions of Python.

Software management, except for cax, will remain mostly unchanged. The collaboration will continue to use CVMFS for software distribution. We are currently reviewing the automatic deployment scheme. 


\section{CONCLUSION}

We have presented the distributed data and job management scheme of the XENON1T experiment. We have shown how an experimental collaboration the size of XENON $(\mathrm{O}(100)$ scientists) can effectively use distributed computing and storage resources using infrastructure and software initially designed and implemented by a much larger scientific collaboration.

\section{ACKNOWLEDGEMENTS}

We gratefully acknowledge support from the National Science Foundation (including Grant No. NSF PHY 1148698), the Swiss National Science Foundation, the German Ministry for Education and Research, Max Planck Gesellschaft, Deutsche Forschungsgemeinschaft, the Netherlands Organisation for Scientific Research (NWO), NLeSC, Weizmann Institute of Science, I-CORE, Pazy-Vatat, Initial Training Network Invisibles (Marie Curie Actions, PITNGA2011-289442), Fundacao para a Ciencia e a Tecnologia, Region des Pays de la Loire, Knut and Alice Wallenberg Foundation, Kavli Foundation, and Instituto Nazionale di Fisica Nucleare. J. Conrad received support from a Wallenberg Academy Fellowship. We are grateful to Laboratori Nazionali del Gran Sasso for hosting and supporting the XENON project

\section{REFERENCES}

[1] 2018. CERN Grid Website. (March 2018). Retrieved March 18, 2018 from https://home.cern/about/computing/grid-system-tiers

[2] 2018. Paramiko Main Website. (2018). Retrieved March 15, 2018 from http: //www.paramiko.org/

[3] 2018. PDC Main Website. (2018). Retrieved March 15, 2018 from https://www pdc.kth.se/sv

[4] 2018. scp man page. (2018). Retrieved March 15, 2018 from http://nixdoc.net/ man-pages/FreeBSD/scp.1.html\#HISTORY

[5] 2018. Tivoli Storage Manager. (2018). Retrieved March 15, 2018 from https://www.ibm.com/developerworks/community/wikis/home?lang=en\# !/wiki/Tivoli\%20Storage\%20Manager/page/Tivoli\%20Storage\%20manager\% 20Version $\% 206 \% 20$ product $\% 20$ family

[6] 2018. UChicago RCC Main Website. (2018). Retrieved March 15, 2018 from https://rcc.uchicago.edu/

[7] 2018. University of Chicago CI Connect. (March 2018). Retrieved March 18, 2018 from https://ci-connect.uchicago.edu/

[8] 2018. Xrootd Main Website. (2018). Retrieved March 15, 2018 from http: //www.xrootd.org/

[9] M. G. Aartsen and et al. 2017. The IceCube Neutrino Observatory: instrumentation and online systems. Fournal of Instrumentation 12 (March 2017), P03012. https: //doi.org/10.1088/1748-0221/12/03/P03012 arXiv:astro-ph.IM/1612.05093

[10] Alex Abramovici and et al. 1992 . LIGO: The Laser Interferometer Gravitational-Wave Observatory. Science 256, 5055 (1992), 325-333. https://doi.org/10.1126/science.256.5055.325 arXiv:http://science.sciencemag.org/content/256/5055/325.full.pdf

[11] M. Ackermann et al. 2017. The Fermi Galactic Center GeV Excess and Implications for Dark Matter. Astrophys. F. 840, 1 (2017), 43. https://doi.org/10.3847/1538-4357/ aa6cab arXiv:astro-ph.HE/1704.03910

[12] P. A. R. Ade et al. 2016. Planck 2015 results. XIII. Cosmological parameters. Astron. Astrophys. 594 (2016), A13. https://doi.org/10.1051/0004-6361/201525830 arXiv:astro-ph.CO/1502.01589

[13] S. Agostinelli and et al. 2003. Geant4 - a simulation toolkit. Nuclear Instruments and Methods in Physics Research Section A: Accelerators, Spectrometers, Detectors and Associated Equipment 506, 3 (2003), 250 - 303. https://doi.org/10.1016/ S0168-9002(03)01368-8

[14] Bryce Allen et al. 2012. Software as a Service for Data Scientists. 55 (02 2012), 81-88.

[15] E. Aprile et al. 2011. Design and performance of the XENON10 dark matter experiment. Astroparticle Physics 34 (April 2011), 679-698. https://doi.org/10. 1016/j.astropartphys.2011.01.006 arXiv:astro-ph.IM/1001.2834

[16] E. Aprile et al. 2016. Physics reach of the XENON1T dark matter experiment. FCAP 1604, 04 (2016), 027. https://doi.org/10.1088/1475-7516/2016/04/027 arXiv:physics.ins-det/1512.07501
[17] aTech Media. 2018. DeployHQ Website. (2018). Retrieved March 15, 2018 from https://www.deployhq.com/

[18] Martin Barisits. 2018. Rucio - Distributed Data Management in ATLAS. (March 2018). Retrieved March 18, 2018 from https: //indico.cern.ch/event/688692/contributions/2836302/attachments/1580448/ 2497243/2018-01-10_CSCS_Presentation_1.pdf

[19] Martin Barisits, Thomas Beermann, Vincent Garonne, Mario Lassnig, and Cedric Serfon. 2018. Rucio GitHub repository. (2018). Retrieved March 15, 2018 from https://github.com/rucio/rucio

[20] L Bauerdick et al. 2012. Using Xrootd to Federate Regional Storage. Fournal of Physics: Conference Series 396, 4 (2012), 042009. http://stacks.iop.org/1742-6596/ $396 / \mathrm{i}=4 / \mathrm{a}=042009$

[21] Jakob Blomer, Predrag Buncic, and Thomas Fuhrmann. 2011. CernVM-FS: Delivering Scientific Software to Globally Distributed Computing Resources. In Proceedings of the First International Workshop on Network-aware Data Management (NDM '11). ACM, New York, NY, USA, 49-56. https://doi.org/10.1145/2110217.2110225

[22] Paul Brenner et al. 2018. Virtual Clusters for Community Computation. (2018). Retrieved March 15, 2018 from https://www.virtualclusters.org/

[23] John Bresnahan, Michael Link, Gaurav Khanna, Zulfikar Imani, Rajkumar Kettimuthu, and Ian Foster. [n. d.]. Globus GridFTP: What's New in 2007 (Invited Paper). ([n. d.]).

[24] P Buncic, C Aguado Sanchez, J Blomer, L Franco, A Harutyunian, P Mato, and Y Yao. 2010. CernVM - a virtual software appliance for LHC applications. Fournal of Physics: Conference Series 219, 4 (2010), 042003. http://stacks.iop.org/1742-6596/ $219 / \mathrm{i}=4 / \mathrm{a}=042003$

[25] The ATLAS Collaboration. 2008. The ATLAS Experiment at the CERN Large Hadron Collider. Fournal of Instrumentation 3, 08 (2008), S08003. http://stacks. iop.org $/ 1748-0221 / 3 / \mathrm{i}=08 / \mathrm{a}=\mathrm{S} 08003$

[26] The CMS Collaboration. 2008. The CMS experiment at the CERN LHC. Fournal of Instrumentation 3, 08 (2008), S08004. http://stacks.iop.org/1748-0221/3/i=08/ $\mathrm{a}=\mathrm{S} 08004$

[27] XENON Collaboration. 2018. XENON GitHub. (March 2018). Retrieved March 18, 2018 from https://github.com/xenon1t

[28] Peter Couvares, Tevfik Kosar, Alain Roy, Jeff Weber, and Kent Wenger. 2007. Workflow Management in Condor. Springer London, London, 357-375. https: //doi.org/10.1007/978-1-84628-757-2_22

[29] Ewa Deelman et al. 2015. Pegasus: a Workflow Management System for Science Automation. Future Generation Computer Systems 46 (2015), 17-35. https: //doi.org/10.1016/j.future.2014.10.008 Funding Acknowledgements: NSF ACI SDCI 0722019 , NSF ACI SI2-SSI 1148515 and NSF OCI-1053575.

[30] Scott Dodelson. 2003. Modern cosmology. Academic Press, San Diego, CA. https://cds.cern.ch/record/1282338

[31] Alvise Dorigo, Peter Elmer, Fabrizio Furano, and Andrew Hanushevsky. 2005. XROOTD/TXNetFile: A Highly Scalable Architecture for Data Access in the ROOT Environment. In Proceedings of the 4th WSEAS International Conference on Telecommunications and Informatics (TELE-INFO'05). World Scientific and Engineering Academy and Society (WSEAS), Stevens Point, Wisconsin, USA, Article 46, 6 pages. http://dl.acm.org/citation.cfm?id=1391157.1391203

[32] I. Foster. 2011. Globus Online: Accelerating and Democratizing Science through Cloud-Based Services. IEEE Internet Computing 15, 3 (May 2011), 70-73. https: //doi.org/10.1109/MIC.2011.64

[33] Martin Hellmich et al. 2014. DPM - efficient storage in diverse environments. Journal of Physics: Conference Series 513, 4 (2014), 042025. http://stacks.iop.org/ $1742-6596 / 513 / \mathrm{i}=4 / \mathrm{a}=042025$

[34] Gerard Jungman, Marc Kamionkowski, and Kim Griest. 1996. Supersymmetric dark matter. Physics Reports 267, 5 (1996), 195 - 373. https://doi.org/10.1016/ 0370-1573(95)00058-5

[35] E. Kolb. 2018. The Early Universe. CRC Press. https://books.google.com/books? id=FwpQDwAAQBAJ

[36] Andrei Kounine. 2012. The Alpha Magnetic Spectrometer on the International Space Station. Int. F. Mod. Phys. E21, 08 (2012), 1230005. https://doi.org/10.1142/ S0218301312300056

[37] D. Kranzlmüller et al. 2010. The European Grid Initiative (EGI). In Remote Instrumentation and Virtual Laboratories. Springer US, Boston, MA, 61-66.

[38] Michael Litzkow, Miron Livny, and Matthew Mutka. 1988. Condor - A Hunter of Idle Workstations. In Proceedings of the 8th International Conference of Distributed Computing Systems.

[39] A P Millar et al. 2014. dCache: Big Data storage for HEP communities and beyond. Journal of Physics: Conference Series 513, 4 (2014), 042033. http://stacks.iop.org/ $1742-6596 / 513 / \mathrm{i}=4 / \mathrm{a}=042033$

[40] Richard L. Moore et al. 2014. Gateways to Discovery: Cyberinfrastructure for the Long Tail of Science. In Proceedings of the 2014 Annual Conference on Extreme Science and Engineering Discovery Environment (XSEDE '14). ACM, New York, NY, USA, Article 39, 8 pages. https://doi.org/10.1145/2616498.2616540

[41] Ruth Pordes et al. 2007. The open science grid. Fournal of Physics: Conference Series 78, 1 (2007), 012057. http://stacks.iop.org/1742-6596/78/i=1/a=012057

[42] Frank Schmuck and Roger Haskin. 2002. GPFS: A Shared-Disk File System for Large Computing Clusters. In Proceedings of the 1st USENIX Conference on File 
and Storage Technologies (FAST '02). USENIX Association, Berkeley, CA, USA, Article 19. http://dl.acm.org/citation.cfm?id=1083323.1083349

[43] I. Sfiligoi, D. C. Bradley, B. Holzman, P. Mhashilkar, S. Padhi, and F. Wurthwein 2009. The Pilot Way to Grid Resources Using glideinWMS. In 2009 WRI World Congress on Computer Science and Information Engineering, Vol. 2. 428-432. https: //doi.org/10.1109/CSIE.2009.950

[44] Douglas Thain, Todd Tannenbaum, and Miron Livny. 2002. Condor and the Grid. In Grid Computing: Making the Global Infrastructure a Reality, Fran Berman, Geoffrey Fox, and Tony Hey (Eds.). John Wiley \& Sons Inc.

[45] Douglas Thain, Todd Tannenbaum, and Miron Livny. 2005. Distributed Computing in Practice: The Condor Experience: Research Articles. Concurr. Comput. Pract. Exper. 17, 2-4 (Feb. 2005), 323-356. https://doi.org/10.1002/cpe.v17:2/4

[46] J. Towns et al. 2014. XSEDE: Accelerating Scientific Discovery. Computing in Science Engineering 16, 5 (Sept 2014), 62-74. https://doi.org/10.1109/MCSE.2014. 80

[47] Rick Wagner, Philip Papadopoulos, Dmitry Mishin, Trevor Cooper, Mahidhar Tatineti, Gregor von Laszewski, Fugang Wang, and Geoffrey C. Fox. 2016. User Managed Virtual Clusters in Comet. In Proceedings of the XSEDE16 Conference on Diversity, Big Data, and Science at Scale (XSEDE16). ACM, New York, NY, USA, Article 24, 8 pages. https://doi.org/10.1145/2949550.2949555

[48] Xenon100 Collaboration, E. Aprile, et al. 2012. The XENON100 dark matter experiment. Astroparticle Physics 35 (April 2012), 573-590. https://doi.org/10. 1016/j.astropartphys.2012.01.003 arXiv:astro-ph.IM/1107.2155 